C, Hawes RH,et al. Primary cysticneoplasms of the pancreas: neoplastic disordersof emerging importance — current state-oftheart and unanswered questions. J Gastrointest Surg. 2003;7:417-28.

3. Choi JY, Kim MJ, Lee JY, Lim JS, Chung JJ, Kim KW, et al.Typical and Atypical Manifestations of Serous Cystadenoma of thePancreas: Imaging Findings with Pathologic Correlation. AJR 2009; 193:136-42.

4. Dewhurst CE, Mortele KJ. Cystic Tumors of the Pancreas: Imaging and Management. Radiol Clin N Am. 2012;50:467-86.

\section{Hilar traumatic neuroma masquerading as Klatskin tumor}

\section{Introduction}

Traumatic neuroma is an exuberant but nonneoplastic proliferation of a nerve that occurs after injury or surgery. ${ }^{1,2}$ They have no specific imaging findings and it is very hard to distinguish a traumatic neuroma from a malignant tumor before surgery. After biliary surgery, neuromas can occur in the cystic duct stump but neuromas involving the bile duct that present as hilar tumors are very rare. We present the case of a traumatic neuroma of the bile duct mimicking hilar cholangiocarcinoma.

\section{Case report}

A 69-year-old woman presented with itching of one-year duration. She had jaundice associated with clay colored stools. There was history of loss of weight and appetite. There was no history of fever, abdominal pain, upper GI bleed and no past history suggestive of hepatic decompensation. There was a past history of laparoscopy converted to open cholecystectomy in 2010 after which she developed a large abdominal swelling which gradually increased in size over time. She was moderately built and nourished.. Icterus was present. There was no pallor, cyanosis, clubbing or edema. There was no supraclavicular lymphadenopathy. Pruritus marks were present over her body. Abdominal examination revealed fullness present in right upper abdomen and a right paramedian scar, which was $12 \mathrm{~cm} \times 10 \mathrm{~cm}$ incisional hernia with large defect present around the scar. Vitals were normal. Abnormal laboratory investigations included raised total bilirubin (2.4 $\mathrm{mg} / \mathrm{dL}$ ), raised alkaline phosphatase (596 IU/L), raised GGTP (808 IU/L) and raised alanine transaminase levels (98 IU/L). Serum CA 19.9 was $136.1 \mathrm{U} / \mathrm{ml}$. Hepatitis serology for $\mathrm{HbsAg}$, anti-HCV, HIV1\&II was negative. Autoimmune serology was negative. On evaluation with CECT, there was evidence of prior surgical intervention and post-operative changes seen in the form of prior cholecystectomy with surgical clips seen at the porta hepatic in close vicinity of the biliary confluence. There was an intra-ductal enhancing soft tissue lesion involving the proximal common duct extending upto the primary biliary confluence with bilobar IHBR dilatation and diagnosis of hilar cholangiocarcinoma was rendered (Figure 1). The patient was taken up for surgery after cardiopulmonary evaluation and clearance. Preoperative diagnosis was hilar cholangiocarcinoma type IIIA, post right portal vein embolization and giant incisional hernia. Procedure performed was staging laparotomy with right hepatectomy with caudate lobe resection and CBD resection. Intraoperative distal bile duct resection margin sent for histopathological frozen section examination was negative for dysplasia and malignancy.

Macroscopically, cut surface of the resected specimen showed a greyish-white firm tumour like lesion composed of nodules measuring together $1.2 \mathrm{~cm}$ and grossly involved proximal common bile duct, common hepatic duct \& left hepatic duct (Figure 2). Histological examination revealed hyperplastic and haphazard proliferation of nerve fibres. These nerve fascicles were well-encapsulated and comprised of small bland Schwann cells and tiny axons, endoneurial cells lying in collagenous stroma (Figures 3 and 4) No evidence of malignancy, atypical mitosis or necrosis was seen in the multiple sections obtained after extensive dissection of the resected biliary system. Immunohistochemical analysis showed S-100 staining of the nerve fibres (Figure 5). Sections from the liver

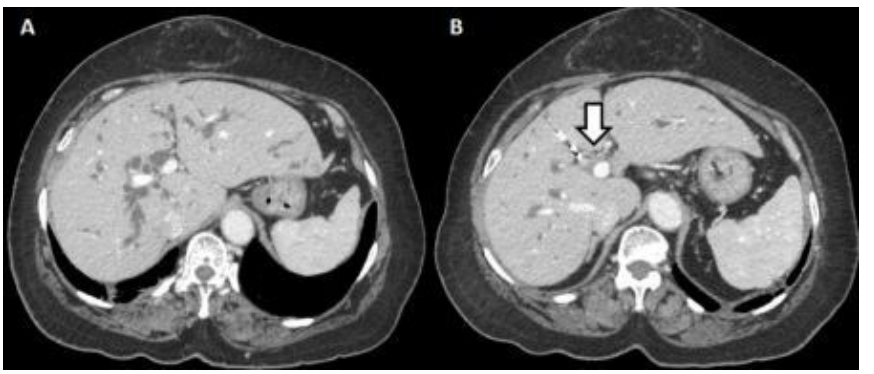

Figure 1: Axial contrast enhanced CT images reveal bilobar intrahepatic biliary dilatation (A) with a subtle ill-defined soft-tissue involving the primary biliary confluence (arrow in B). The lesion is seen in close proximity with the post-cholecystectomy metallic clips. 


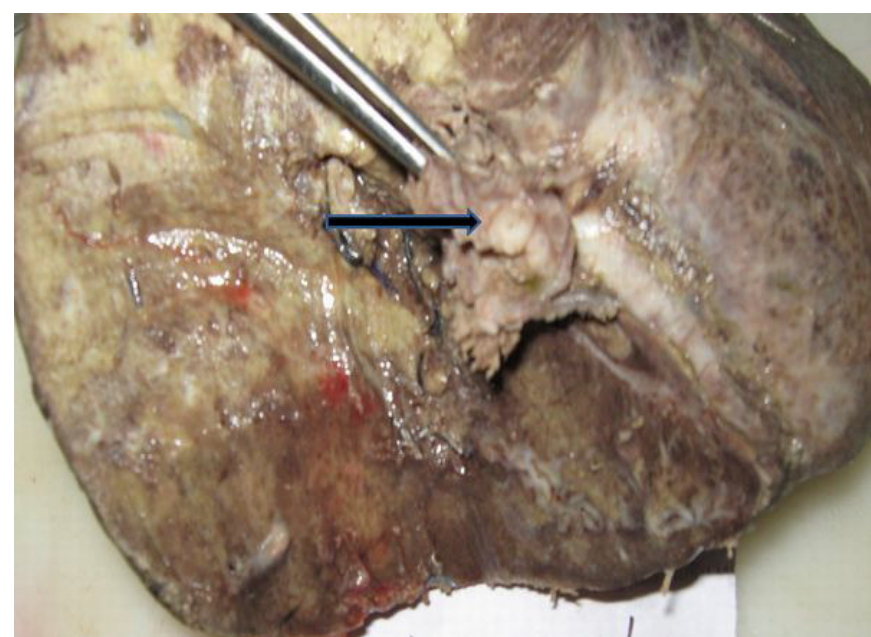

Figure 2: Right hepatectomy gross specimen showing hilar biliary apparatus showing Grey-white small variable sized nodules involving proximal common bile duct, common hepatic duct \& left hepatic duct

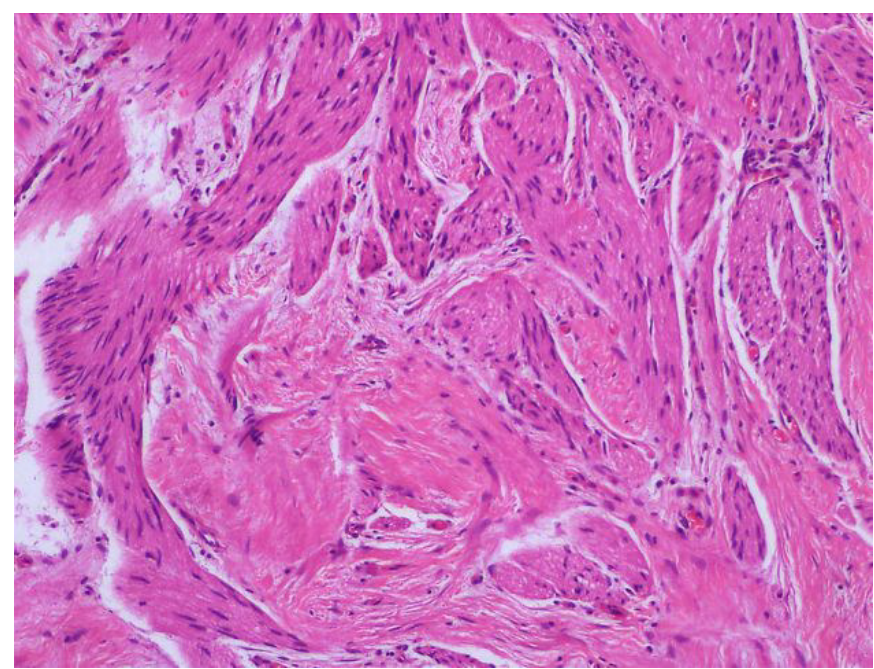

Figure 3: Hilar lesion showing disorganized nerve fascicles with intervening fibrocollagenous tissue (HE, x200).

showed evidence of embolization of portal vein branches with presence of foreign body material walled off with granulation tissue and foreign body type of granulomas and giant cell reaction. Histopathological diagnosis of 'Hilar Traumatic Neuroma' was rendered. Patient is doing well on follow-up of 6 months.

\section{Discussion}

Traumatic neuromas arise from Schwann sheath cells located at the ends of severed or injured nerves. It is a non-neoplastic disorganized proliferation of axons, Schwann cells, and perineurial cells in a fibrocollagenous stroma and affects nerves that are encased in Schwann cells. Neuroma formation is thought to be precipitated by posttraumatic nerve cell growth

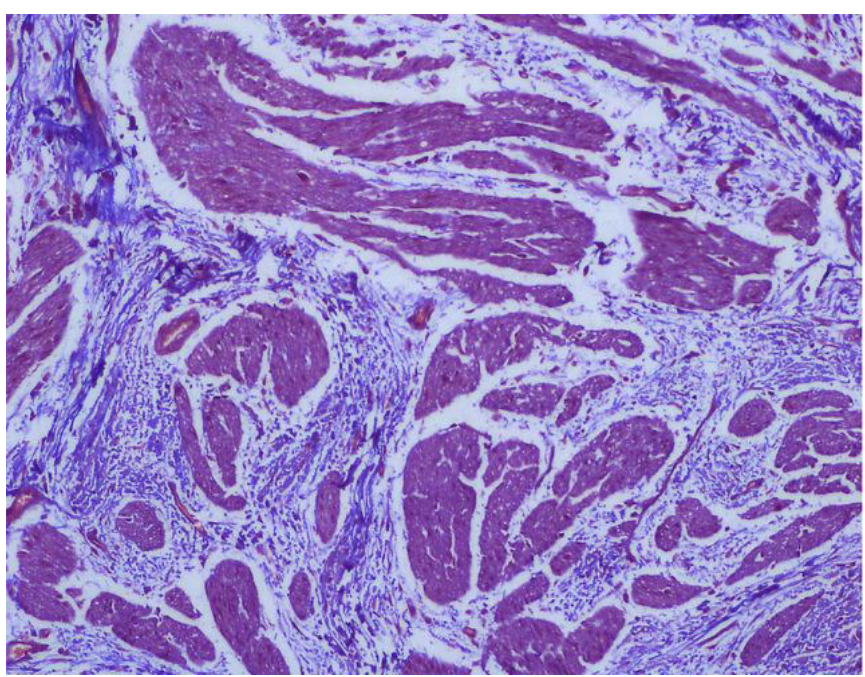

Figure 4: Hilar lesion with haphazard arrangement of nerve tissue (red) and fibrocollagneous tissue (blue) (Masson trichrome, $\mathrm{x} 200$ )

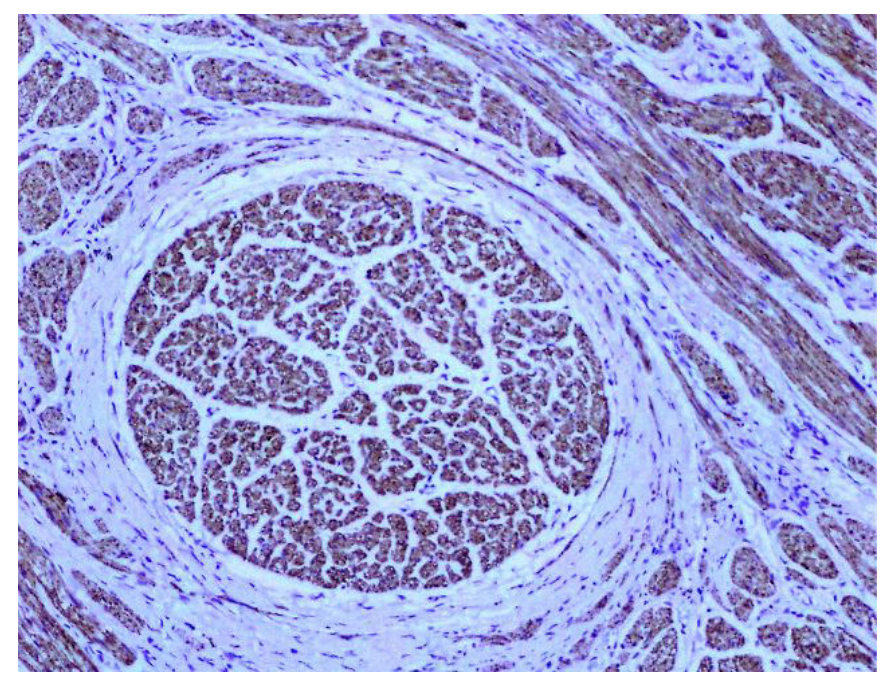

Figure 5: Immunohistochemical staining of nerve fascicles with S$100(\mathrm{x} 100)$

after surgery and such nerve hypertrophy in response to injury is similar to that seen with extremity amputations. ${ }^{1,2,3}$

Neuroma of the biliary tree was first described in 1928 by Husseinoff. ${ }^{4}$ Most commonly, a traumatic neuroma occurs in the cystic duct stump after cholecystectomy because the common bile duct is surrounded by an abundant nerve supply. ${ }^{5}$ Bile duct neuroma was demonstrated in up to $10 \%$ of post cholecystectomy patients at autopsy in one study. ${ }^{6}$ Common bile duct exploration has also been associated with neuroma formation. Traumatic neuromas (TN) of the biliary tree causing strictures have also been reported after liver transplantation. ${ }^{6}$ Hilar traumatic neuroma is very rare.

Preoperative and imaging diagnosis of traumatic neuroma is difficult however possibility of traumatic neuroma should always be considered in the differential diagnosis of patients 
suspected of hilar cholangiocarcinoma who had previous cholecystectomy.

ARCHANA RASTOGI ${ }^{1}$, CHHAGAN BIHARI ${ }^{1}$, ANKUR ARORA ${ }^{2}$,

PIYUSH KUMAR SINHA ${ }^{3}$, TUSHAR KANTI CHATTOPADHYAY ${ }^{3}$ KISHORE GSB ${ }^{3}$

Correspondence: Dr Archana Rastogi Department of Pathology $y^{l}$ Department of Radiology ${ }^{2}$ and Department of Hepatopancreaticobiliary Surgery ${ }^{3}$ Institute of Liver and Biliary Sciences D-1,Vasant Kunj, New Delhi-110070, India. Email:drarchanarastogi@gmail.com

\section{References}

1. Hotta T, Yahuhito K, Katsutoshi T, Naka T, Johata K, Sahara $\mathrm{M}$, et al. A traumatic neuroma of the bile duct: a case report. Hepatogastroenterology 2004;51:39-42.

2. Pickens A, Vickers S, Brown K, Reddy VV, Thompson JA. An unusual etiology of biliary hilar obstruction and the potential role of acidic fibroblast growth factor in the development of a biliary neuroma. Am Surgeon. 1999;65:47-51.

3. Zeff R, Pfeffer R, Adams P, Ruoff M. Reoperation for amputation neuroma of the cystic duct. Am J Surg 1976;131:369-70.

4. Husseinoff D. Ueber einem Fall von Wucherung des Nervengewebes nach wiederholten Operationen der Gallenga: nge. Zbl Allg Path. 1928;43:344-8.

5. Schfiroff BGP, Hilton JW. Surgical anatomy of the choledochal nerves. Arch Surg. 1950;60:944-52.

6. Herrera L, Martino E, Rodríguez-Sanjuán JC, Castillo J, Casafont F, González F, et al. Traumatic neuroma of extrahepatic bile ducts after orthotopic liver transplantation. Transplant Proc. 2009;41:1054-6.

\section{Recurrent diarrhea: an unusual case of lupus enteritis}

\section{Introduction}

Gastrointestinal manifestations of SLE are diverse and can involve the entire gastrointestinal system. ${ }^{1}$ A syndrome of abdominal pain and diarrhoea in SLE can be due to pancreatitis, serositis, protein-losing enteropathy, gluten enteropathy, intestinal pseudo-obstruction, infection, and lupus enteritis/vasculitis. Lupus enteritis is defined as either vasculitis or inflammation of the small-bowel, with supportive image and/or biopsy findings (BILAG 2004). ${ }^{2}$ Lupus enteritis is a rare complication of SLE, presumably mediated by immunecomplex deposition and complement activation, with subsequent submucosal edema. Abdominal computed tomography (CT) is the most useful diagnostic tool and is key in leading to the correct diagnosis of lupus enteritis. We describe the case of a female patient presenting with recurrent intractable nausea, vomiting and diarrhoea in the absence of previously diagnosed SLE.

\section{Case history}

A 20 year-old-female patient was admitted to our hospital with severe nausea, vomiting and intractable watery diarrhea of one months' duration (not responding to antibiotics, probiotics, fasting or antimotility agents) associated with abdominal pain, diminished urine output, and increased urinary frequency; Examination revealed pallor, dehydration, mild ascites and abdominal tenderness. She had three similar self-limiting episodes of nausea, vomiting and diarrhoea in the past two years, each requiring hospitalization and improved transiently with intravenous antibiotics and fluids over 3-4 week period. A history of occasional low grade fever was present in last two years. She had iron deficiency anaemia with a haemoglobin level of $7.2 \mathrm{gm} \%$ and normal liver and renal function tests.

24 hour urinary protein estimation revealed proteinuria of $1.8 \mathrm{~g} /$ day. Ultrasonography of whole abdomen revealed bilateral hydroureteronephrosis with edematous bladder wall and ascites. A contrast enhanced CT scan of the abdomen and pelvis showed diffuse circumferential bowel wall thickening in the small intestine (Target sign-Figure 1) in addition to ultrasonographic findings. Work up for auto immune parameters revealed antinuclear antibody- 1:160 (mixed homogenous and speckled pattern), anti-dsDNA antibody-91 $\mathrm{u} / \mathrm{ml}$ (normal <55), complement C3-83.4 mg/dl (normal range 90180), lupus anticoagulant and anticardiolipin antibodies were negative. Upper GI endoscopy revealed mild erythema of gastric corpus and D2 biopsy was normal. Colonoscopy revealed diffuse mild erythema and edema of entire colonic mucosa with no ulcerations. In view of proteinuria, a renal biopsy was done. Histopathologic examination revealed membranous 